Report no. 01/11

\title{
A family of finite difference schemes for the convection-diffusion equation in two dimensions
}

\author{
E. Sousa \& I.J. Sobey \\ Oxford University \\ Numerical Analysis Group
}

The construction of finite difference schemes in two dimensions is more ambiguous than in one dimension. This ambiguity arises because different combinations of local nodal values are equally able to model local behaviour with the same order of accuracy.

In this paper we outline an evolutionary operator for the two dimensional convection-diffusion problem in an unbounded domain and use it as the source for obtaining a family of second order (Lax-Wendroff) schemes and third-order (Quickest) schemes not yet studied in the literature. Additionally we study in detail the stability of those second-order and third-order schemes, a crucial property for convergence of numerical schemes, using the von Neumann method.

Subject classifications: AMS(MOS): 65L12, 65L20

Key words and phrases: finite differences, convection-diffusion, von Neumann method, numerical stability

Oxford University Computing Laboratory

Numerical Analysis Group

Wolfson Building

Parks Road

Oxford, England OX1 3QD

E-mail: sousa@comlab.oxford.ac.uk

June, 2001 


\section{Introduction}

At the heart of computational fluid dynamics, including computation of laminar and turbulent flow and of heat and mass transfer, lies a deceptively simple balance of convection and diffusion. Despite its simplicity, this balance is very difficult to simulate without artificial effects such as increased dispersion or oscillations degrading the solution fidelity. These effects take on increased importance in two and three dimensional flows because of difficulty in resolving all possible length scales. Great advances in simulating convection-diffusion have occurred in the last two decades and it is now possible to devise schemes of arbitrary accuracy for constant velocity convection in an unbounded domain. We have an understanding of how schemes which previously might be classed finite element or finite difference can be reconciled in a relatively general evolutionary operator framework (see Morton and Sobey [9] and Morton [10]).

In this paper we deduce a new family of Lax-Wendroff schemes and Quickest schemes by using an evolutionary operator in an unbounded domain for the two dimensional convection-diffusion problem. The one dimensional Lax-Wendroff scheme is due to Lax and Wendroff [6] and the Quickest scheme was introduced by Leonard [7] as an alternative to central differencing convection or to upwinding differencing convection.

The Lax-Wendroff schemes are a class of schemes which have attained considerable stature in theoretical studies of difference schemes. The essential property of the Lax-Wendroff schemes lies in the combination of time and space-centred discretisations. Their popularity is due to their second-order accuracy and simplicity, although their behaviour around discontinuities is not fully satisfactory.

The Quickest scheme was first generalised in two dimensions by Davis and Moore [2]. When generalising the method they ignored some of the cross-derivatives and that reduced the temporal accuracy of the scheme.

Although the Lax-Wendroff and Quickest schemes are unique for the one dimensional case, they have many variants in the two-dimensional case. Their ambiguity in two dimensions is connected with the fact that different combinations of local nodal values are equally able to model local behaviour. In this paper, we describe the analytical solution for a two dimensional convection-diffusion problem and use it as the source for obtaining Lax-Wendroff and Quickest schemes not yet studied in the literature. The new Quickest schemes are expected to be more accurate in time than the Quickest scheme deduced by Davis and Moore [2], since we take into account the cross-derivatives. Additionally we study in detail the stability of those Lax-Wendroff and Quickest schemes, a crucial property for convergence of numerical schemes.

To analyse the practical stability of the numerical schemes we use von Neumann analysis since we are considering a problem in an unbounded domain. We observe that interesting differences occur between the stability regions of the different numerical schemes. For a clear visualisation of the stability regions we plot the sufficient and necessary stability conditions in a three-dimensional space, in which the coordinates involve the convective coefficients and the diffusion.

Stability of finite difference schemes has been widely described in the literature. Two important books on stability analysis of difference methods are the classical book 
by Richtmyer and Morton [11] and the more recent book by Gustafsson et al [3]. The latter concentrates its attention in the normal mode analysis.

Some of the work on stability analysis for finite difference schemes for the convectiondiffusion equation using von Neumann method was done by Beckers [1] for a scheme in three dimensions, Hindmarsh et al [4] for a multidimensional central scheme, Kwok and Tan [5] for leap-frog-type finite difference schemes, Siemieniuch and Gladwell [12] for central and upwind schemes, Verwer and Sommeijer [15] in relation with an odd-evenline hopscotch method, and Wesseling [14] for a fourth-order central scheme.

\section{Analytic Solution}

Morton and Sobey [9] have deduced schemes using the exact solution of a one dimensional convection-diffusion problem. Applying the same idea, in this section we deduce the analytic solution for a two dimensional convection-diffusion problem and use that as the source for obtaining the finite difference schemes.

Consider the convection-diffusion equation with coefficient $D>0$ :

$$
\frac{\partial u}{\partial t}(x, y, t)+V \frac{\partial u}{\partial x}(x, y, t)+W \frac{\partial u}{\partial y}(x, y, t)=D\left(\frac{\partial^{2} u}{\partial x^{2}}+\frac{\partial^{2} u}{\partial y^{2}}\right)(x, y, t)
$$

and the initial condition

$$
u(x, y, 0)=u_{0}(x, y) .
$$

The diffusion coefficient is taken to be positive since a negative coefficient is a physical impossibility. We take a two-dimensional Fourier transform, denoted $\hat{u}(l, m)$ where $l$ and $m$ are transform variables in the $x$ and $y$ directions. Thus

$$
\hat{u}(l, m, t)=u_{0}(l, m) \mathrm{e}^{-\left[D l^{2}+D m^{2}\right] t+\mathrm{i}[V l+W m] t} .
$$

Writing the inversion of this transform as a convolution integral,

$$
u(x, y, t)=\frac{1}{\pi} \int_{-\infty}^{+\infty} \int_{-\infty}^{+\infty} u_{0}(x-V t+2 \sqrt{D t} \xi, y-W t+2 \sqrt{D t} \tau) \mathrm{e}^{-\xi^{2}-\tau^{2}} d \xi d \tau .
$$

This is a two-dimensional evolution operator. In a similar manner, to the onedimensional case, it defines a Green's function $G(x, y, \Delta t)$ which gives the evolution over a single time-step:

$$
u\left(x, y, t_{n}+\Delta t\right)=\frac{1}{\pi} \int_{-\infty}^{+\infty} \int_{-\infty}^{+\infty} u\left(\xi, \eta, t_{n}\right) G(x-\xi, y-\eta, \Delta t) d \xi d \eta
$$

where

$$
G(s, p, t)=\frac{1}{4 D t \pi} \mathrm{e}^{-(s-V t)^{2} / 4 D t} \mathrm{e}^{-(p-W t)^{2} / 4 D t} .
$$

In the next section we deduce finite difference schemes for an unbounded domain using the evolutionary operator (2.4), following the same procedure as in Morton and Sobey [9] for one dimension. 


\section{Finite differences schemes}

With the general framework described in the previous section we can now obtain finite difference schemes by interpolation on a uniform mesh.

We denote $U_{j k}^{n}$ the approximations to the values $u\left(x_{j}, y_{k}, t_{n}\right)$ at the mesh points

$$
\left(x_{j}, y_{k}\right)=(j \Delta x, k \Delta y), \quad j, k=0, \pm 1, \pm 2, \ldots
$$

We use the usual operators, central, second difference, backward and forward respectively:

$$
\begin{aligned}
\Delta_{x 0} U_{j k} & =\frac{U_{j+1 k}-U_{j-1 k}}{2}, \\
\delta_{x}^{2} U_{j k} & =\frac{U_{j+1 k}-2 U_{j k}+U_{j-1 k}}{2}, \\
\Delta_{x-} U_{j k} & =U_{j k}-U_{j-1 k} \\
\Delta_{x+} U_{j k} & =U_{j+1 k}-U_{j k} .
\end{aligned}
$$

The operators $\Delta_{y 0}, \delta_{y}^{2}, \Delta_{y-}, \Delta_{y+}$ are defined similarly.

We choose uniform space steps $\Delta x$ and $\Delta y$ and a time step $\Delta t$. We also define the important quantities $\nu_{x}, \nu_{y}, \mu_{x}$ and $\mu_{y}$, that we are using in what follows,

$$
\begin{array}{ll}
\nu_{x}=\frac{V \Delta t}{\Delta x}, & \nu_{y}=\frac{W \Delta t}{\Delta y}, \\
\mu_{x}=\frac{D \Delta t}{\Delta x^{2}}, & \mu_{y}=\frac{D \Delta t}{\Delta y^{2}} .
\end{array}
$$

In the next sections we deduce a family of Lax-Wendroff schemes and a family of Quickest schemes in two dimensions. Firstly we deduce the schemes based in a quadratic and cubic polynomial interpolation of the function $u\left(\xi, \eta, t_{n}\right)$ that appears in $(2.4)$. Secondly we also deduce schemes by using a Taylor approximation of order two and three, of the same function.

The generalisation of finite difference schemes for a convection diffusion equation to multidimensions is not just the sum of the individual one-dimension contributions, since the simple addition of individual finite differences in $x$ and $y$ without appropriate cross terms can lead to a basic instability.

\subsection{Polynomial approximation}

In this section we obtain finite difference schemes by approximating $u\left(x, y, t_{n}\right)$ in $(2.4)$ by a local polynomial around the point $\left(x_{j}, y_{k}\right)$, namely

$$
p_{j k}(x, y)=\sum_{r=0}^{K} \sum_{s=0}^{K} b_{r s}\left(x-x_{j}\right)^{r}\left(y-y_{k}\right)^{s} .
$$


Using the exact evolutionary operator, then the approximation $U_{j k}^{n+1}$ is given by

$$
\begin{aligned}
& U_{j k}^{n+1}=\sum_{r, s=0}^{K} \frac{b_{r s}}{\pi} \int_{-\infty}^{+\infty}(-V \Delta t+2 \sqrt{D \Delta t} \xi)^{r} \mathrm{e}^{-\xi^{2}} d \xi \\
& \int_{-\infty}^{+\infty}(-W \Delta t+2 \sqrt{D \Delta t} \tau)^{s} \mathrm{e}^{-\tau^{2}} d \tau .
\end{aligned}
$$

If the power terms are expanded then all the integrals can be determined and then we can write,

$$
\begin{aligned}
U_{j k}^{n+1}= & b_{00}-b_{10} V \Delta t-b_{01} W \Delta t+b_{11} V W(\Delta t)^{2} \\
& +b_{20}\left(2 D \Delta t+V^{2}(\Delta t)^{2}\right)+b_{02}\left(2 D \Delta t+W^{2}(\Delta t)^{2}\right) \\
& -b_{30}\left(6 D V(\Delta t)^{2}+V^{3}(\Delta t)^{3}\right)-b_{03}\left(6 D W(\Delta t)^{2}+W^{3}(\Delta t)^{3}\right) \\
& -b_{21} W \Delta t\left(2 D \Delta t+V^{2}(\Delta t)^{2}\right)-b_{12} V \Delta t\left(2 D \Delta t+W^{2}(\Delta t)^{2}\right) \\
& +\ldots
\end{aligned}
$$

Within this formula we obtain second and third order finite difference schemes by using quadratic interpolation or cubic interpolation.

\section{Quadratic polynomial interpolation}

If we use a quadratic interpolation we need to choose six interpolation points to determine the six coefficients $b_{00}, b_{01}, b_{10}, b_{20}, b_{11}, b_{02}$. We obtain a different method for each choice of points. Any nodal value will have eight neighbouring points so we need to choose six points from the nine points (nodal point plus eight neighbours). We choose the points, so that they form a five-point star around $\left(x_{j}, y_{k}\right)$ and the sixth point is selected according to the direction of the velocities $V$ and $W$. For instance assume that $V$ and $W$ are positive. We choose the six interpolation point to be $\left(x_{j-1}, y_{k-1}\right)$. Then we have

$$
\begin{aligned}
b_{00}=U_{j k}^{n} & b_{10}=\frac{\Delta_{x 0} U_{j k}^{n}}{\Delta x} & b_{01} & =\frac{\Delta_{y 0} U_{j k}^{n}}{\Delta y} \\
b_{11}=\frac{\Delta_{x-} \Delta_{y-} U_{j k}^{n}}{\Delta x \Delta y} & b_{02}=\frac{1}{2} \frac{\delta_{x}^{2} U_{j k}^{n}}{\Delta x^{2}} & b_{20} & =\frac{1}{2} \frac{\delta_{y}^{2} U_{j k}^{n}}{\Delta y^{2}}
\end{aligned}
$$

This gives the formula,

$$
\begin{aligned}
U_{j k}^{n+1}= & {\left[1-\left(\nu_{x} \Delta_{x 0}+\nu_{y} \Delta_{y 0}\right)+\left(\frac{1}{2} \nu_{x}^{2}+\mu_{x}\right) \delta_{x}^{2}\right.} \\
& \left.+\left(\frac{1}{2} \nu_{y}^{2}+\mu_{y}\right) \delta_{y}^{2}+\nu_{x} \nu_{y} \Delta_{x-} \Delta_{y-}\right] U_{j k}^{n} .
\end{aligned}
$$

We call this scheme Polynomial Lax-Wendroff. 


\begin{tabular}{|ccccc|}
\hline & $V \geq 0, W \geq 0$ & $V \geq 0, W \leq 0$ & $V \leq 0, W \geq 0$ & $V \leq 0, W \leq 0$ \\
\hline & & & & \\
$b_{11}$ & $\Delta_{x-} \Delta_{y-}$ & $\Delta_{x-} \Delta_{y+}$ & $\Delta_{x+} \Delta_{y-}$ & $\Delta_{x+} \Delta_{y+}$ \\
\hline
\end{tabular}

Table 1: Polynomial Lax-Wendroff scheme.

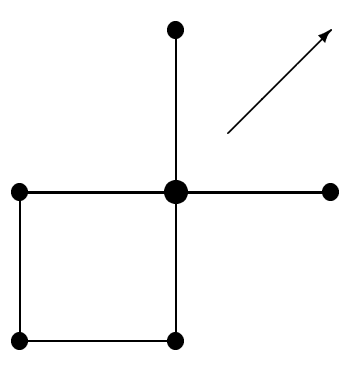

(a)

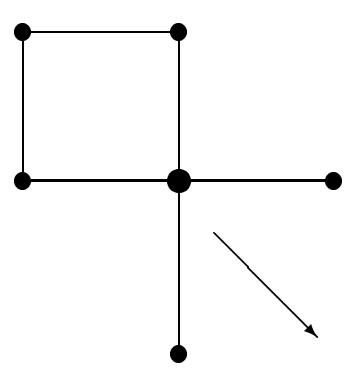

(c)

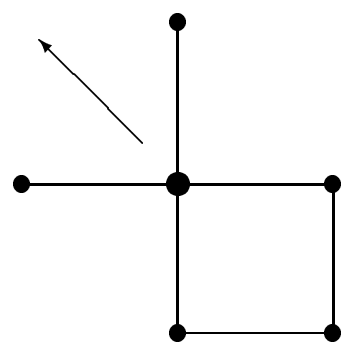

(b)

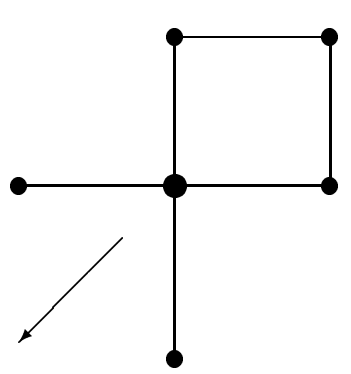

(d)

Figure 1: Computational molecule for the Polynomial Lax-Wendroff scheme for the different signs of the velocities: (a) $V$ and $W$ positive; (b) $V$ negative and $W$ positive; (c) $V$ positive and $W$ negative; (d) $V$ and $W$ negative. The larger circle denotes the central node and arrows show typical flow directions.

Clearly there are three other configurations depending on various combinations of the sign of $V$ and $W$. For $V$ negative and $W$ positive, we choose the sixth point as $\left(x_{j+1}, y_{k-1}\right)$. If $V$ is positive and $W$ negative, we consider the point $\left(x_{j-1}, y_{k+1}\right)$ and for $V$ and $W$ negative we choose the point $\left(x_{j-1}, y_{k+1}\right)$. The different possibilities can give us a different coefficient $b_{11}$. We describe in the table 1 how the operators that define the coefficient $b_{11}$ change according to the changes of the signs of the velocities.

We illustrate the different computational molecules in figure 1 together with the flow directions.

\section{Cubic polynomial interpolation}

Next we turn to cubic interpolation. One advantage of using high-order methods is 


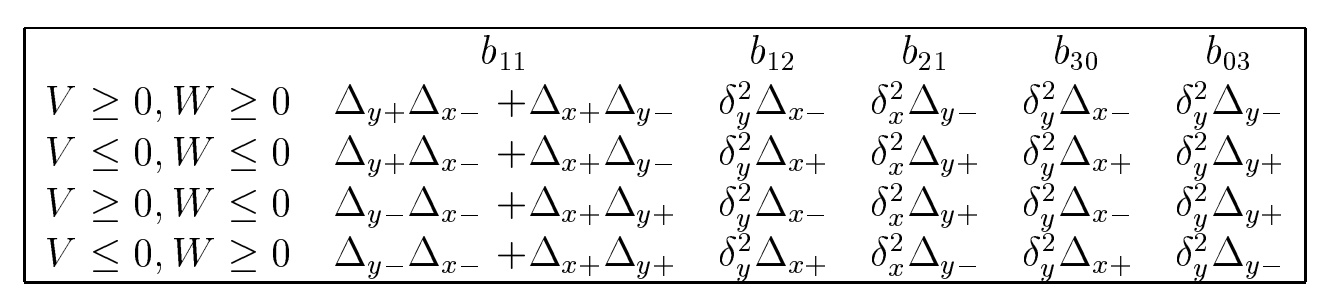

Table 2: Polynomial Quickest scheme

that numerical diffusion and dispersion errors are relatively smaller than in low order methods.

The procedure is illustrated for two-dimensional flow with velocity $V$ and $W$ positive as in the previous case. We need to use 10 points to carry out this interpolation. Using the 10 points $U_{j-2 k}, U_{j-1 k-1}, U_{j-1 k}, U_{j-1 k+1}, U_{j k-2}, U_{j k-1}, U_{j k}, U_{j k+1}, U_{j+1 k-1}$ and $U_{j+1 k}$ to evaluate $b_{r s}, r=0,1,2,3 ; s=0,1,2,3$ we find

$$
\begin{aligned}
b_{00} & =U_{j k} & b_{10} & =\frac{\Delta_{x 0} U_{j k}}{\Delta x}-\frac{\delta_{x}^{2} \Delta_{x-} U_{j k}}{6 \Delta x} \\
b_{20} & =\frac{\delta_{x}^{2} U_{j k}}{2 \Delta x^{2}} & b_{30} & =\frac{\delta_{x}^{2} \Delta_{x-} U_{j k}}{6 \Delta x^{3}} \\
b_{01} & =\frac{\Delta_{y 0} U_{j k}}{\Delta y}-\frac{\delta_{y}^{2} \Delta_{y-} U_{j k}}{6 \Delta y} & b_{21} & =\frac{\delta_{x}^{2} \Delta_{y-} U_{j k}}{2 \Delta x^{2} \Delta y} \\
b_{02} & =\frac{\delta_{y}^{2} U_{j k}}{2 \Delta y^{2}} & b_{03} & =\frac{\delta_{y}^{2} \Delta_{y-} U_{j k}}{6 \Delta y^{3}} \\
b_{12} & =\frac{\delta_{y}^{2} \Delta_{x-} U_{j k}}{2 \Delta y^{2} \Delta x} & b_{11} & =\frac{\Delta_{y+} \Delta_{x-} U_{j k}}{2 \Delta x \Delta y}+\frac{\Delta_{x+} \Delta_{y-} U_{j k}}{2 \Delta x \Delta y}
\end{aligned}
$$

Now we can write the scheme,

$$
\begin{aligned}
U_{j k}^{n+1}= & U_{j k}^{n}-\nu_{x} \Delta_{x 0} U_{j k}^{n}-\nu_{y} \Delta_{y 0} U_{j k}^{n} \\
& +\left(\frac{1}{2} \nu_{x}^{2}+\mu_{x}\right) \delta_{x}^{2} U_{j k}^{n}+\left(\frac{1}{2} \nu_{y}^{2}+\mu_{y}\right) \delta_{y}^{2} U_{j k}^{n} \\
& +\frac{1}{2} \nu_{x} \nu_{y} \Delta_{y+} \Delta_{x-} U_{j k}^{n}+\frac{1}{2} \nu_{x} \nu_{y} \Delta_{x+} \Delta_{y-} U_{j k}^{n} \\
& +\frac{1}{6} \nu_{x}\left(1-\nu_{x}^{2}-6 \mu_{x}\right) \delta_{x}^{2} \Delta_{x-} U_{j k}^{n}+\frac{1}{6} \nu_{y}\left(1-\nu_{y}^{2}-6 \mu_{y}\right) \delta_{y}^{2} \Delta_{y-} U_{j k}^{n} \\
& -\nu_{y}\left(\mu_{x}+\frac{1}{2} \nu_{x}^{2}\right) \delta_{x}^{2} \Delta_{y-} U_{j k}^{n}-\nu_{x}\left(\mu_{y}+\frac{1}{2} \nu_{y}^{2}\right) \delta_{y}^{2} \Delta_{x-} U_{j k}^{n} .
\end{aligned}
$$

This scheme is called Polynomial Quickest scheme. As with the Lax-Wendroff schemes, we can change the choice of the mesh points, depending on the direction of the velocities. The changes that occur in the scheme (3.2) according to the sign of the velocities involve changes in the coefficients $b_{11}, b_{12}, b_{21}, b_{30}$ and $b_{03}$ that we describe in table 2 .

We illustrate the different computational molecules in figure 2 together with the flow directions. 


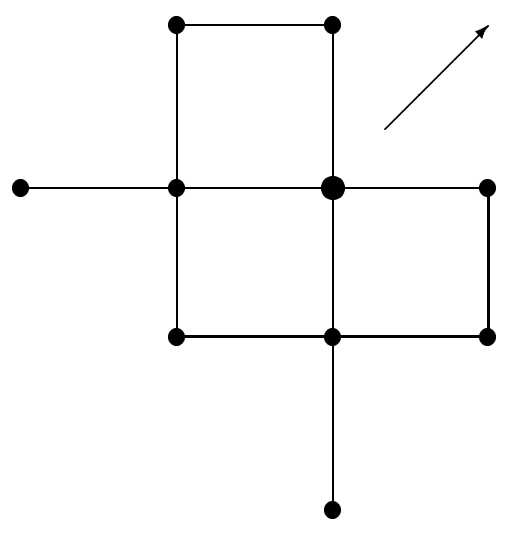

(a)

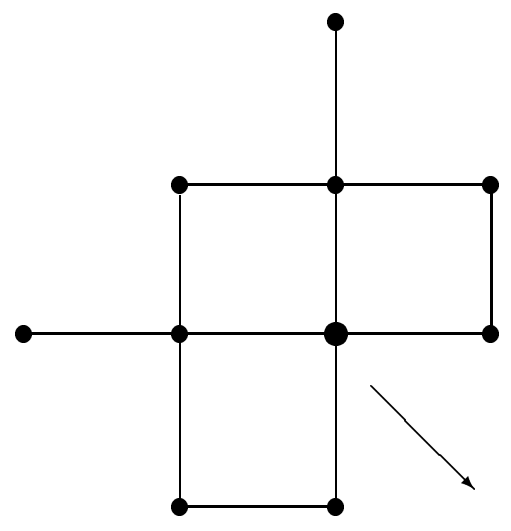

(c)

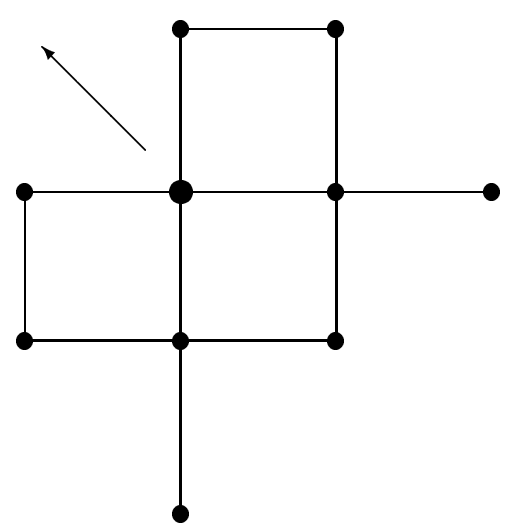

(b)

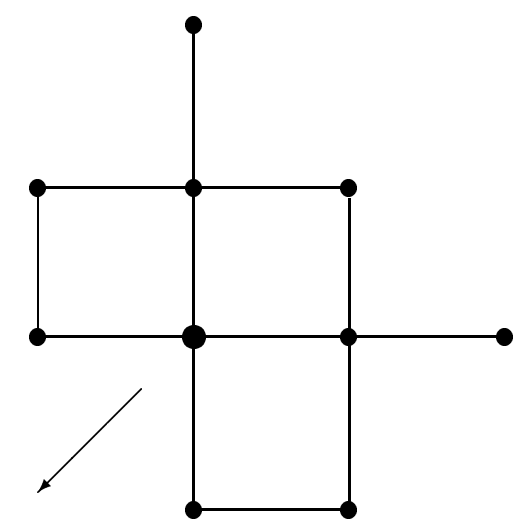

(d)

Figure 2: Computational molecule for the Polynomial Quickest scheme for the different signs of the velocities: (a) $V$ and $W$ positive; (b) $V$ negative and $W$ positive; (c) $V$ positive and $W$ negative; (d) $V$ and $W$ negative. The larger circle denotes the central node and predominant flow directions are shown by arrows. 


\subsection{Taylor approximation}

In the previous section we considered a local polynomial interpolation of some selected points in a neighbourhood of $\left(x_{j}, y_{k}\right)$. Since the local interpolation requires only a small number of neighbouring points, we used the flow directions to choose which neighbouring points to use. Now we use an alternative idea and approximate $u\left(x, y, t_{n}\right)$ by a truncated Taylor series around $\left(x_{j}, y_{k}\right)$ :

$$
t_{j k}(x, y)=\sum_{r=0}^{K} \sum_{s=0}^{K} b_{r s}\left(x-x_{j}\right)^{r}\left(y-y_{k}\right)^{s},
$$

where $b_{r s}=\frac{u_{x^{r} y^{s}}}{r ! s !}$. Using the evolutionary operator as in the previous section, depending on the order of the expansion we can obtain the numerical schemes described below.

\section{Second order Taylor expansion}

For the second order accurate Taylor expansion the $b_{r s}$ coefficients are:

$$
\begin{array}{lll}
b_{00}=U_{j k} & b_{10}=u_{x}:=\frac{\Delta_{x 0} U_{j k}}{\Delta x} \\
b_{01}=u_{y}:=\frac{\Delta_{y 0} U_{j k}}{\Delta y}-\frac{\delta_{y}^{2} \Delta_{y-} U_{j k}}{6 \Delta y} & b_{20}=\frac{1}{2} u_{x x}:=\frac{\delta_{x}^{2} U_{j k}}{2 \Delta x^{2}} \\
b_{02}=\frac{1}{2} u_{y y}:=\frac{\delta_{y}^{2} U_{j k}}{2 \Delta y^{2}} & b_{11}=u_{x y}:=\frac{\Delta_{x 0} \Delta_{y 0} U_{j k}}{\Delta x \Delta y}
\end{array}
$$

This scheme uses a 9 points stencil and the numerical method has the form:

$$
\begin{aligned}
U_{j k}^{n+1}= & {\left[1-\left(\nu_{x} \Delta_{x 0}+\nu_{y} \Delta_{y 0}\right)+\left(\frac{1}{2} \nu_{x}^{2}+\mu_{x}\right) \delta_{x}^{2}\right.} \\
& \left.+\left(\frac{1}{2} \nu_{y}^{2}+\mu_{y}\right) \delta_{y}^{2}+\nu_{x} \nu_{y} \Delta_{x 0} \Delta_{y 0}\right] U_{j k}^{n} .
\end{aligned}
$$

We call this the Taylor Lax-Wendroff scheme. This formula is illustrated by the computational molecule in figure 3 . This molecule can be used independently of the directions of the velocities.

\section{Third order Taylor expansion}

When we derive different Lax-Wendroff schemes they differ because of the way we discretise the mixed derivatives. The same is true in the case of the two different Quickest schemes. We discretise the mixed derivatives $u_{x y}, u_{x y y}$ and $u_{y y x}$ in a different way in both schemes or, to put it differently, we choose in a different way the coefficients $b_{11}, b_{12}$ and $b_{21}$.

For the third order accurate Taylor expansion, taking in consideration that $V$ and $W$ are positive, we use the 11 point stencil, $U_{j-2 k}, U_{j-1 k-1}, U_{j-1 k} U_{j-1 k+1}, U_{j k-2}, U_{j k-1}$, 


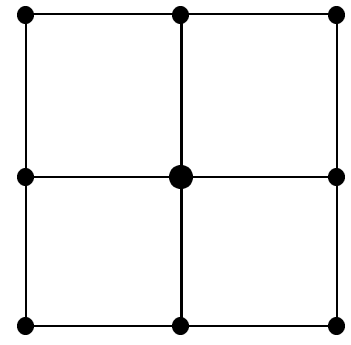

Figure 3: Computational molecule for the Taylor Lax-Wendroff scheme. The larger circle denotes the central node.

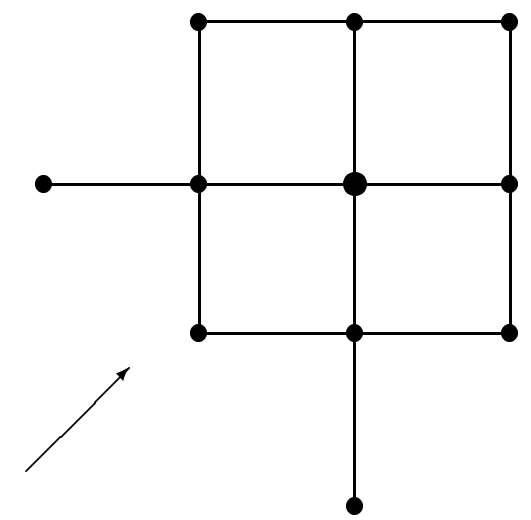

Figure 4: Computational molecule for the Taylor Quickest scheme. The larger circle denotes the central node and predominant flow direction is shown by arrow. 


\begin{tabular}{|ccccc|}
\hline & $V \geq 0, W \geq 0$ & $V \geq 0, W \leq 0$ & $V \leq 0, W \geq 0$ & $V \leq 0, W \leq 0$ \\
$b_{30}$ & $\delta_{x}^{2} \Delta_{x-}$ & $\delta_{x}^{2} \Delta_{x-}$ & $\delta_{x}^{2} \Delta_{x+}$ & $\delta_{x}^{2} \Delta_{x+}$ \\
$b_{03}$ & $\delta_{y}^{2} \Delta_{y-}$ & $\delta_{y}^{2} \Delta_{y+}$ & $\delta_{y}^{2} \Delta_{y-}$ & $\delta_{y}^{2} \Delta_{y+}$ \\
\hline
\end{tabular}

Table 3: Taylor Quickest scheme.

$U_{j k}, U_{j k+1} U_{j+1 k-1}, U_{j+1 k}$, and $U_{j+1 k+1}$. It follows:

$$
\begin{aligned}
b_{00} & =U_{j k} & b_{10} & =u_{x}:=\frac{\Delta_{x 0} U_{j k}}{\Delta x}-\frac{\delta_{x}^{2} \Delta_{x-} U_{j k}}{6 \Delta x} \\
b_{20} & =\frac{1}{2} u_{x x}:=\frac{\delta_{x}^{2} U_{j k}}{2 \Delta x^{2}} & b_{30} & =\frac{1}{6} u_{x x x}:=\frac{\delta_{x}^{2} \Delta_{x-} U_{j k}}{6 \Delta x^{3}} \\
b_{01} & =u_{y}:=\frac{\Delta_{y 0} U_{j k}}{\Delta y}-\frac{\delta_{y}^{2} \Delta_{y-} U_{j k}}{6 \Delta y} & b_{21} & =\frac{1}{2} u_{x x y}:=\frac{\delta_{x}^{2} \Delta_{y 0} U_{j k}}{2 \Delta x^{2} \Delta y} \\
b_{02} & =\frac{1}{2} u_{y y}:=\frac{\delta_{y}^{2} U_{j k}}{2 \Delta y^{2}} & b_{03} & =\frac{1}{6} u_{y y y}:=\frac{\delta_{y}^{2} \Delta_{y-} U_{j k}}{6 \Delta y^{3}} \\
b_{12} & =\frac{1}{2} u_{y y x}:=\frac{\delta_{y}^{2} \Delta_{x 0} U_{j k}}{2 \Delta y^{2} \Delta x} & b_{11} & =u_{x y}:=\frac{\Delta_{x 0} \Delta_{y 0} U_{j k}}{\Delta x \Delta y}
\end{aligned}
$$

We have the numerical method,

$$
\begin{aligned}
U_{j k}^{n+1}= & U_{j k}^{n}-\nu_{x} \Delta_{x 0} U_{j k}^{n}-\nu_{y} \Delta_{y 0} U_{j k}^{n} \\
& +\left(\frac{1}{2} \nu_{x}^{2}+\mu_{x}\right) \delta_{x}^{2} U_{j k}^{n}+\left(\frac{1}{2} \nu_{y}^{2}+\mu_{y}\right) \delta_{y}^{2} U_{j k}^{n} \\
& +\nu_{x} \nu_{y} \Delta_{x 0} \Delta_{y 0} U_{j k}^{n} \\
& +\frac{1}{6} \nu_{x}\left(1-\nu_{x}^{2}-6 \mu_{x}\right) \delta_{x}^{2} \Delta_{x-} U_{j k}^{n}+\frac{1}{6} \nu_{y}\left(1-\nu_{y}^{2}-6 \mu_{y}\right) \delta_{y}^{2} \Delta_{y-} U_{j k}^{n} \\
& -\nu_{y}\left(\mu_{x}+\frac{1}{2} \nu_{x}^{2}\right) \delta_{x}^{2} \Delta_{y 0} U_{j k}^{n}-\nu_{x}\left(\mu_{y}+\frac{1}{2} \nu_{y}^{2}\right) \delta_{y}^{2} \Delta_{x 0} U_{j k}^{n} .
\end{aligned}
$$

Similarly to the previous schemes, we call this scheme the Taylor Quickest scheme. This formula is illustrated by the computational molecule in figure 4 . The choice of the mesh points, to approximate the third derivatives, depends on the direction of the velocities and it affects the values of the coefficients $b_{30}$ and $b_{03}$. The changes are shown in table 3 .

\subsection{Summary of the schemes}

To conclude this section we summarise the numerical schemes derived here and whose stability we analyse in the following sections (in this summary we assume both velocities positive). 
- Polynomial Lax-Wendroff scheme (see figure 1)

$$
U_{j k}^{n+1}=\left[1+\mathcal{P}_{x}+\mathcal{P}_{y}+\nu_{x} \nu_{y} \Delta_{x-} \Delta_{y-}\right] U_{j k}^{n} .
$$

- Taylor Lax-Wendroff scheme (see figure 3)

$$
U_{j k}^{n+1}=\left[1+\mathcal{P}_{x}+\mathcal{P}_{y}+\nu_{x} \nu_{y} \Delta_{x 0} \Delta_{y 0}\right] U_{j k}^{n}
$$

where

$$
\begin{aligned}
& \mathcal{P}_{x}=-\nu_{x} \Delta_{x 0}+\left(\frac{1}{2} \nu_{x}^{2}+\mu_{x}\right) \delta_{x}^{2} \\
& \mathcal{P}_{y}=-\nu_{y} \Delta_{y 0}+\left(\frac{1}{2} \nu_{y}^{2}+\mu_{y}\right) \delta_{y}^{2}
\end{aligned}
$$

- Polynomial Quickest scheme (see figure 2)

$$
\begin{aligned}
U_{j k}^{n+1}= & {\left[1+\mathcal{Q}_{x}+\mathcal{Q}_{y}+\frac{1}{2} \nu_{x} \nu_{y}\left(\Delta_{y+} \Delta_{x-}+\Delta_{x+} \Delta_{y-}\right)\right.} \\
& \left.-\nu_{y}\left(\mu_{x}+\frac{1}{2} \nu_{x}^{2}\right) \delta_{x}^{2} \Delta_{y-}-\nu_{x}\left(\mu_{y}+\frac{1}{2} \nu_{y}^{2}\right) \delta_{y}^{2} \Delta_{x-}\right] U_{j k}^{n}
\end{aligned}
$$

- Taylor Quickest scheme (see figure 4)

$$
\begin{aligned}
U_{j k}^{n+1}= & {\left[1+\mathcal{Q}_{x}+\mathcal{Q}_{y}+\nu_{x} \nu_{y} \Delta_{x 0} \Delta_{y 0}\right.} \\
& \left.-\nu_{y}\left(\mu_{x}+\frac{1}{2} \nu_{x}^{2}\right) \delta_{x}^{2} \Delta_{y 0}-\nu_{x}\left(\mu_{y}+\frac{1}{2} \nu_{y}^{2}\right) \delta_{y}^{2} \Delta_{x 0}\right] U_{j k}^{n} .
\end{aligned}
$$

where

$$
\begin{aligned}
\mathcal{Q}_{x} & =\mathcal{P}_{x}+\frac{1}{6} \nu_{x}\left(1-\nu_{x}^{2}-6 \mu_{x}\right) \delta_{x}^{2} \Delta_{x-} \\
\mathcal{Q}_{y} & =\mathcal{P}_{y}+\frac{1}{6} \nu_{y}\left(1-\nu_{y}^{2}-6 \mu_{y}\right) \delta_{y}^{2} \Delta_{y-}
\end{aligned}
$$

In the next section we use the von Neumann method to analyse the stability region of these schemes. 


\section{Von Neumann stability analysis}

The von Neumann analysis in two dimensions is a straightforward generalisation of the one dimensional case. The discrete Fourier decomposition in two dimensions consists of the decomposition of the function into a Fourier series as

$$
U_{j k}^{n}=\sum_{\xi_{x}, \xi_{y}} \kappa^{n} \mathrm{e}^{\mathrm{i} \xi_{x} j \Delta x} \mathrm{e}^{\mathrm{i} \xi_{y} k \Delta y}
$$

where the range $\xi_{x}, \xi_{y}$ is defined separately for each direction, as in the one dimensional case. The amplification factor is given by $\kappa$. The products $\xi_{x} \Delta x$ and $\xi_{y} \Delta y$ are often represented as a phase angle, namely:

$$
\theta_{x}=\xi_{x} \Delta x, \quad \theta_{y}=\xi_{y} \Delta y
$$

To obtain a von Neumann stability condition we insert the singular component

$$
\kappa^{n} \mathrm{e}^{\mathrm{i} j \theta_{x}} \mathrm{e}^{\mathrm{i} k \theta_{y}}
$$

into the discretised scheme. The amplification factor is said to satisfy the von Neumann condition if there is a constant $K$ such that

$$
\left|\kappa\left(\theta_{x}, \theta_{y}\right)\right| \leq 1+K \Delta t, \quad \forall \theta_{x}, \theta_{y} \in[0,2 \pi] .
$$

As in the one dimensional case, in practice we use the stronger condition

$$
\left|\kappa\left(\theta_{x}, \theta_{y}\right)\right| \leq 1, \quad \forall \theta_{x}, \theta_{y} \in[0,2 \pi]
$$

and the discrete scheme that meets this condition, we refer to it as von Neumann stable. This has been called practical stability by Richtmyer and Morton [11] or strict stability by other authors. In some cases condition (4.1) allows numerical modes to grow exponentially in time for finite values of $\Delta t$. Therefore, the practical, or strict, stability condition (4.2) is recommended in order to prevent numerical modes from growing faster than the physical modes of the differential equation.

For our finite difference schemes we derive mostly analytical necessary conditions. Nevertheless we plot conditions, determined numerically, that are sufficient and necessary for stability.

First we analyse the Fourier stability analysis for the convective problem, this is for $D=0$, although we are mainly interested in problems with diffusion. Afterwards we study the fully convective and diffusive discrete scheme.

\subsection{Practical stability regions for $D=0$}

In this section we consider the convective problem and analyse the different stability regions obtained for the Lax-Wendroff schemes and the Quickest schemes. We consider first the Lax-Wendroff schemes. 


\section{Lax-Wendroff schemes}

The next result is related with the scheme (3.1) derived using a quadratic polynomial interpolation and called Polynomial Lax-Wendroff scheme. Although we were not able to prove the sufficient and necessary condition plotted in figure $5 \mathrm{a}$ for the scheme (3.1), we prove analytical necessary conditions.

Lemma 4.1 Necessary conditions for the stability of the Polynomial Lax-Wendroff scheme, when $D=0, \nu_{x} \geq 0$ and $\nu_{y} \geq 0$ are:

$$
\begin{aligned}
& \left(\nu_{x}-\nu_{y}\right) \leq 1, \\
& \nu_{x} \leq 1, \quad \nu_{y} \leq 1 .
\end{aligned}
$$

Proof: The amplification factor for the method (3.1), when $D=0$ verifies the equality:

$$
\begin{aligned}
\left|\kappa\left(\theta_{x}, \theta_{y}\right)\right|^{2}= & {\left[1-\nu_{x}^{2}\left(1-\cos \theta_{x}\right)-\nu_{y}^{2}\left(1-\cos \theta_{y}\right)\right.} \\
& \left.+\nu_{x} \nu_{y}\left(\left(1-\cos \theta_{x}\right)\left(1-\cos \theta_{y}\right)-\sin \theta_{x} \sin \theta_{y}\right)\right]^{2} \\
& +\left[-\nu_{x} \sin \theta_{x}-\nu_{y} \sin \theta_{y}\right. \\
& \left.+\nu_{x} \nu_{y}\left(\sin \theta_{x}\left(1-\cos \theta_{y}\right)+\sin \theta_{y}\left(1-\cos \theta_{x}\right)\right)\right]^{2}
\end{aligned}
$$

In particular,

$$
\begin{aligned}
|\kappa(\pi, \pi)| & =\left|1-2 \nu_{x}^{2}-2 \nu_{y}^{2}+4 \nu_{x} \nu_{y}\right|, \\
& =\left|1-2\left(\nu_{x}-\nu_{y}\right)^{2}\right| \\
|\kappa(0, \pi)| & =\left|1-2 \nu_{y}^{2}\right| \\
|\kappa(\pi, 0)| & =\left|1-2 \nu_{x}^{2}\right| .
\end{aligned}
$$

If we have $|\kappa(\pi, \pi)| \leq 1,|\kappa(0, \pi)| \leq 1$ and $|\kappa(\pi, 0)| \leq 1$ then (4.3) and (4.4) are verified.

The stability of the scheme (3.3), here called Taylor Lax-Wendroff scheme, was already studied in literature for $D=0$. The next result is due to Turkel [13].

Theorem 4.2 For $D=0$ the scheme (3.3) is stable iff

$$
\left|\nu_{x}\right|^{2 / 3}+\left|\nu_{y}\right|^{2 / 3} \leq 1
$$

Proof: See Turkel [13].

The condition (4.5) of the Theorem 4.2 is plotted in figure $5 \mathrm{~b}$. 


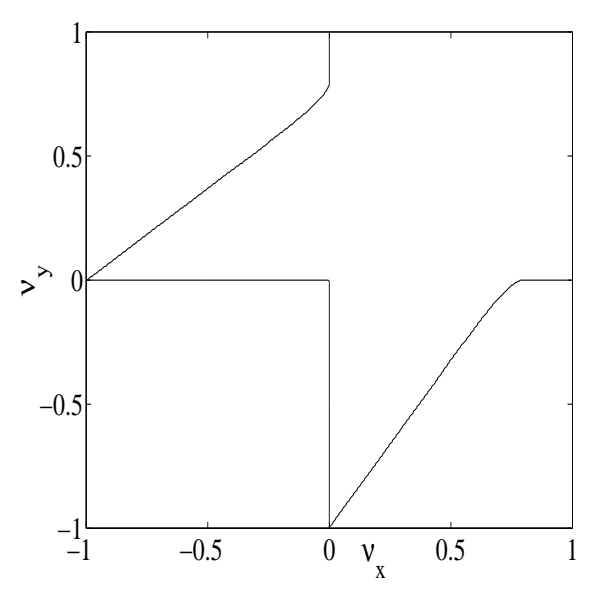

(a)

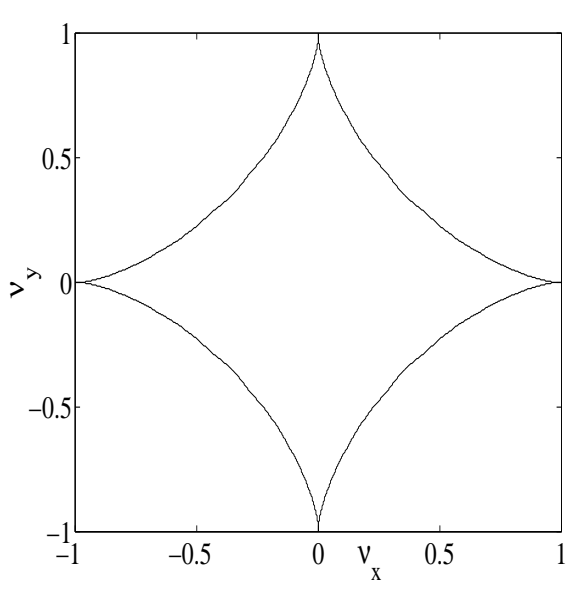

(b)

Figure 5: (a) Stability region for the Polynomial Lax-Wendroff scheme for $\mu_{x}=\mu_{y}=0$ (the scheme was deduced for both velocities positive); (b) Stability region for the Taylor LaxWendroff scheme for $\mu_{x}=\mu_{y}=0$, deduced independently of the signs of both velocities.

\section{Quickest schemes}

First we consider the Polynomial Quickest scheme and we present a necessary condition for this scheme. This necessary condition is also a sufficient condition although we do not prove it analytically. We plot numerically the sufficient and necessary stability condition for the Polynomial Quickest scheme in figure 6a.

Lemma 4.3 If the Polynomial Quickest scheme (3.2) is stable, for $D=0,0 \leq \nu_{x} \leq 1$ and $0 \leq \nu_{y} \leq 1$ then

$$
\nu_{x}+\nu_{y} \leq 1
$$

Proof: The amplification factor for the Polynomial Quickest scheme is given by:

$$
\begin{aligned}
\kappa\left(\theta_{x}, \theta_{y}\right)= & 1-\mathrm{i} \nu_{x} \sin \theta_{x}-\mathrm{i} \nu_{y} \sin \theta_{y}+\nu_{x}^{2}\left(-1+\cos \theta_{x}\right)+\nu_{y}^{2}\left(-1+\cos \theta_{y}\right) \\
& -\nu_{x} \nu_{y}\left(\left(1-\cos \theta_{x}\right)\left(1-\cos \theta_{y}\right)+\sin \theta_{x} \sin \theta_{y}\right) \\
& +\frac{1}{6} \nu_{x}\left(1-\nu_{x}^{2}\right)\left[-2\left(1-\cos \theta_{x}\right)^{2}+2 \mathrm{i} \sin \theta_{x}\left(-1+\cos \theta_{x}\right)\right] \\
& +\frac{1}{6} \nu_{y}\left(1-\nu_{y}^{2}\right)\left[-2\left(1-\cos \theta_{y}\right)^{2}+2 \mathrm{i} \sin \theta_{y}\left(-1+\cos \theta_{y}\right)\right] \\
& -\frac{1}{2} \nu_{y} \nu_{x}^{2}\left[-2\left(1-\cos \theta_{y}\right)\left(1-\cos \theta_{x}\right)+2 \mathrm{i} \sin \theta_{y}\left(-1+\cos \theta_{x}\right)\right] \\
& -\frac{1}{2} \nu_{x} \nu_{y}^{2}\left[-2\left(1-\cos \theta_{x}\right)\left(1-\cos \theta_{y}\right)+2 \mathrm{i} \sin \theta_{x}\left(-1+\cos \theta_{y}\right)\right] .
\end{aligned}
$$

In particular we have,

$$
\kappa(\pi, \pi)=1-2 \nu_{x}^{2}-2 \nu_{y}^{2}-4 \nu_{x} \nu_{y}-\frac{4}{3} \nu_{x}\left(1-\nu_{x}^{2}\right)
$$




$$
\begin{aligned}
& -\frac{4}{3} \nu_{y}\left(1-\nu_{y}^{2}\right)+4 \nu_{x} \nu_{y}^{2}+4 \nu_{y} \nu_{x}^{2} \\
= & 1-2\left(\nu_{x}+\nu_{y}\right)^{2}-\frac{4}{3}\left(\nu_{x}+\nu_{y}\right)+\frac{4}{3}\left(\nu_{x}+\nu_{y}\right)^{3} .
\end{aligned}
$$

To have $|\kappa(\pi, \pi)| \leq 1$ implies $\nu_{x}+\nu_{y} \leq 1$ or $\nu_{x}+\nu_{y} \geq 3 / 2$. From this fact we obtain (4.6).

The next lemma is a necessary condition for the Taylor Quickest scheme. We plot the necessary and sufficient conditions for this scheme in figure $6 \mathrm{~b}$.

Lemma 4.4 If the Taylor Quickest scheme (3.4) is stable, for $D=0,0 \leq \nu_{x} \leq 1$ and $0 \leq \nu_{y} \leq 1$ then

$$
\nu_{x}+\nu_{y} \leq 1
$$

Proof: The amplification factor for the Taylor Quickest scheme is

$$
\begin{aligned}
\kappa\left(\theta_{x}, \theta_{y}\right)= & 1-\mathrm{i} \nu_{x} \sin \theta_{x}-\mathrm{i} \nu_{y} \sin \theta_{y} \\
& +\nu_{x}^{2}\left(-1+\cos \theta_{x}\right)+\nu_{y}^{2}\left(-1+\cos \theta_{y}\right) \\
& -\nu_{x} \nu_{y} \sin \theta_{x} \sin \theta_{y} \\
& -\frac{1}{3} \nu_{x}\left(1-\nu_{x}^{2}\right)\left[\left(1-\cos \theta_{x}\right)^{2}+\mathrm{i} \sin \theta_{x}\left(1-\cos \theta_{x}\right)\right] \\
& -\frac{1}{3} \nu_{y}\left(1-\nu_{y}^{2}\right)\left[\left(1-\cos \theta_{y}\right)^{2}+\mathrm{i} \sin \theta_{y}\left(1-\cos \theta_{y}\right)\right] \\
& +\nu_{y} \nu_{x}^{2} \mathrm{i} \sin \theta_{y}\left(1-\cos \theta_{x}\right)+\nu_{x} \nu_{y}^{2} \mathrm{i} \sin \theta_{x}\left(1-\cos \theta_{y}\right) .
\end{aligned}
$$

As in the previous cases we consider in particular,

$$
\begin{aligned}
\kappa(\pi, \pi)= & 1-2 \nu_{x}^{2}-2 \nu_{y}^{2}-\frac{4}{3} \nu_{x}\left(1-\nu_{x}^{2}\right)-\frac{4}{3} \nu_{y}\left(1-\nu_{y}^{2}\right) \\
= & 1-2\left[\left(\nu_{x}+\nu_{y}\right)^{2}+\frac{2}{3}\left(\nu_{x}+\nu_{y}\right)-\frac{2}{3}\left(\nu_{x}+\nu_{y}\right)^{3}\right. \\
& \left.-2 \nu_{x} \nu_{y}\left(1-\nu_{x}-\nu_{y}\right)\right]
\end{aligned}
$$

and if $|\kappa(\pi, \pi)| \leq 1$ then we have (4.7) .

In this section we assumed $D=0$. In the next section we analyse the stability regions for the schemes considered in this section but with the diffusive coefficient $D>0$.

\subsection{Practical stability regions for $D>0$}

In this section we consider the parabolic problem that is simultaneously convective and diffusive. We analyse the stability analytically, obtaining mainly necessary conditions. We plot numerically the sufficient and necessary stability regions in the three dimensional space $\left(\nu_{x}, \nu_{y}, \mu\right)$, where for simplicity we assume $\mu=\mu_{x}=\mu_{y}$. 


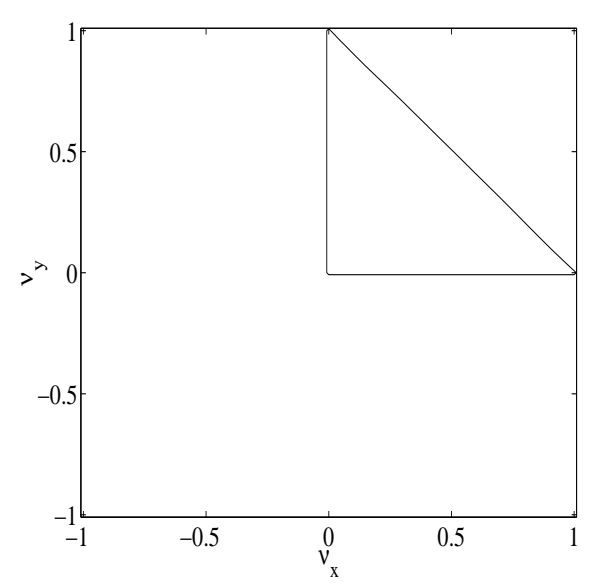

(a)

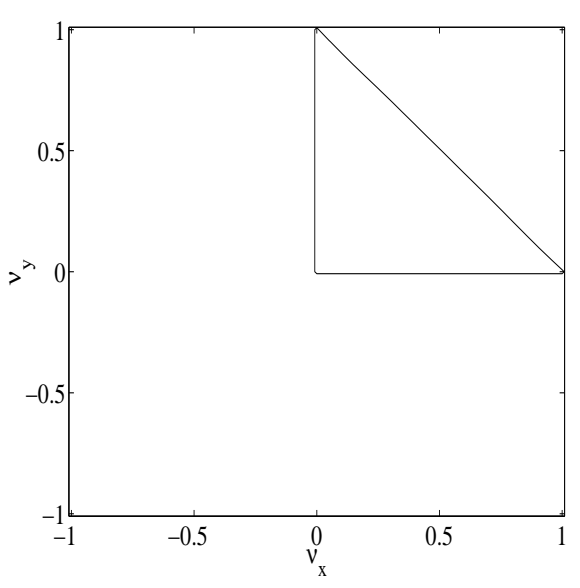

(b)

Figure 6: (a) Von Neumann stability region for the Polynomial Quickest scheme for $\mu_{x}=$ $\mu_{y}=0$, deduced when both velocities are considered positive and consequently $\nu_{x}$ and $\nu_{y}$ are positive; (b) Von Neumann stability region for the Taylor Quickest scheme for $\mu_{x}=\mu_{y}=0$, deduced when both velocities are considered positive and consequently $\nu_{x}$ and $\nu_{y}$ are positive

A finite difference scheme for the two-dimensional convection diffusion equation (2.1), which is quite well known in the literature is the central scheme:

$$
U_{j k}^{n+1}=U_{j k}^{n}-\nu_{x} \Delta_{x 0} U_{j k}^{n}-\nu_{y} \Delta_{y 0} U_{j k}^{n}+\mu_{x} \delta_{x}^{2} U_{j k}^{n}+\mu_{y} \delta_{y}^{2} U_{j k}^{n} .
$$

The von Neumann stability analysis of this scheme was studied by Hindmarsh and Gresho [4] for a multidimensional problem.

We show the stability region of the central scheme in order to compare it with the Lax-Wendroff schemes and the Quickest schemes that we are studying. The von Neumann necessary and sufficient conditions for stability, for the central scheme (4.8), are given in the next theorem due to Hindmarsh and Gresho [4]. They are also plotted in figure 7 , for $\mu=\mu_{x}=\mu_{y}$.

Theorem 4.5 The scheme (4.8) is stable, in the von Neumann sense, if and only if

$$
\begin{aligned}
2 \mu_{x}+2 \mu_{y} & \leq 1 \\
\frac{\nu_{x}^{2}}{2 \mu_{x}}+\frac{\nu_{y}^{2}}{2 \mu_{y}} & \leq 1 .
\end{aligned}
$$

Proof: See Hindmarsh and Gresho [4].

In figure $7 \mathrm{~b}$ and figure $7 \mathrm{c}$ we show the projections of figure $7 \mathrm{a}$ in two different planes to give us a more accurate idea of the stability region. In particular, note that the region of stability in the $\left(\nu_{x}, \nu_{y}\right)$-plane decreases rapidly as $\mu \rightarrow 0$. 


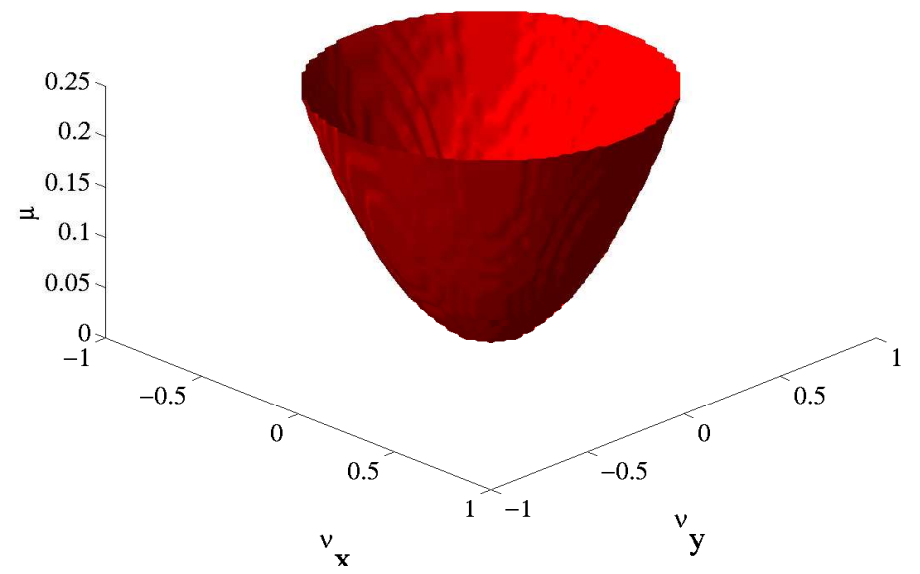

(a)

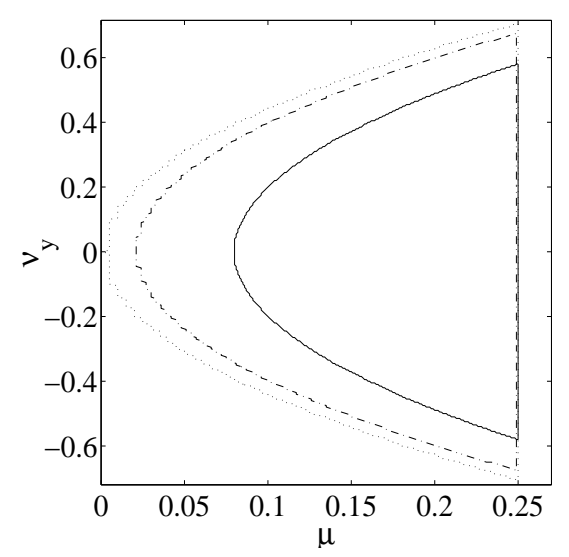

(b)

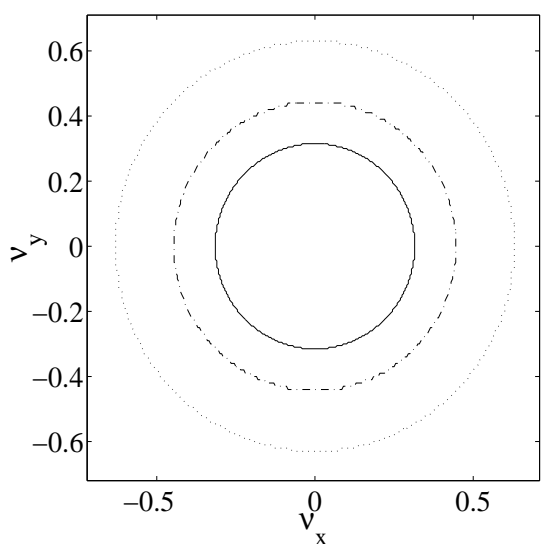

(c)

Figure 7: (a) von Neumann stability analysis for the central scheme. The scheme is stable inside the parabolized shape; (b) projection of the figure (a) on the plane $\mu \circ \nu_{y}: \nu_{x}=0(\cdots)$; $\nu_{x}=0.2(-\cdot-) ; \nu_{x}=0.4(-) ;\left(\right.$ c) projection of the figure (a) on the plane $\nu_{x} \circ \nu_{y}: \mu=0.05(-)$; $\mu=0.1(-\cdot-) ; \mu=0.2(\cdots)$. 
For the next finite difference schemes we derive mostly analytical necessary conditions. Nevertheless we plot sufficient and necessary conditions for stability determined numerically.

\section{Lax-Wendroff schemes}

We start to analyse the Polynomial Lax-Wendroff scheme by giving some necessary conditions for the stability of this scheme.

Lemma 4.6 Necessary conditions for the Polynomial Lax-Wendroff scheme (3.1) to be stable are

$$
\begin{aligned}
2\left(\mu_{x}+\mu_{y}\right) & \leq 1 \\
\left(\nu_{x}-\nu_{y}\right)^{2} & \leq 1-2\left(\mu_{x}+\mu_{y}\right) .
\end{aligned}
$$

Proof: The square of the modulus of the amplification factor for the Polynomial LaxWendroff is,

$$
\begin{aligned}
\left|\kappa\left(\theta_{x}, \theta_{y}\right)\right|^{2}= & {\left[1-\left(\nu_{x}^{2}+2 \mu_{x}\right)\left(1-\cos \theta_{x}\right)-\left(\nu_{y}^{2}+2 \mu_{y}\right)\left(1-\cos \theta_{y}\right)\right.} \\
& \left.+\nu_{x} \nu_{y}\left(\left(1-\cos \theta_{x}\right)\left(1-\cos \theta_{y}\right)-\sin \theta_{x} \sin \theta_{y}\right)\right]^{2} \\
& +\left[-\nu_{x} \sin \theta_{x}-\nu_{y} \sin \theta_{y}\right. \\
& \left.+\nu_{x} \nu_{y}\left(\sin \theta_{x}\left(1-\cos \theta_{y}\right)+\sin \theta_{y}\left(1-\cos \theta_{x}\right)\right)\right]^{2} .
\end{aligned}
$$

For the limiting case $\theta_{x} \rightarrow 0$ and $\theta_{y} \rightarrow 0$ with $\left|\theta_{x}\right| \leq \theta$ and $\left|\theta_{y}\right| \leq \theta$, we can write

$$
\begin{aligned}
\left|\kappa\left(\theta_{x}, \theta_{y}\right)\right|^{2}= & {\left[1-\left(\nu_{x}^{2}+2 \mu_{x}\right) \frac{\theta_{x}^{2}}{2}-\left(\nu_{y}^{2}+2 \mu_{y}\right) \frac{\theta_{y}^{2}}{2}\right.} \\
& \left.+\nu_{x} \nu_{y}\left(\frac{\theta_{x}^{2}}{2} \frac{\theta_{y}^{2}}{2}-\theta_{x} \theta_{y}\right)+O\left(\theta^{4}\right)\right]^{2} \\
& +\left[-\nu_{x} \theta_{x}-\nu_{y} \theta_{y}+\nu_{x} \nu_{y}\left(\theta_{x} \frac{\theta_{y}^{2}}{2}+\theta_{y} \frac{\theta_{x}^{2}}{2}\right)+O\left(\theta^{3}\right)\right]^{2} .
\end{aligned}
$$

After some algebraic calculations we obtain,

$$
\begin{aligned}
\left|\kappa\left(\theta_{x}, \theta_{y}\right)\right|^{2}= & 1-\left(\nu_{x}^{2}+2 \mu_{x}\right) \theta_{x}^{2}-\left(\nu_{y}^{2}+2 \mu_{y}\right) \theta_{y}^{2}-2 \nu_{x} \nu_{y} \theta_{x} \theta_{y} \\
& +\left(\nu_{x} \theta_{x}+\nu_{y} \theta_{y}\right)^{2}+O\left(\theta^{4}\right) \\
= & 1-\left(2 \mu_{x}+2 \mu_{y}\right)+O\left(\theta^{4}\right) .
\end{aligned}
$$

In order to have $\left|\kappa\left(\theta_{x}, \theta_{y}\right)\right| \leq 1$ for all $\theta_{x}, \theta_{y}$ we need to have (4.11). For the particular case $\theta_{x}=\theta_{y}=\pi$ we have,

$$
|\kappa(\pi, \pi)|=1-2\left(\nu_{x}^{2}+2 \mu_{x}\right)-2\left(\nu_{y}^{2}+2 \mu_{y}\right)+4 \nu_{x} \nu_{y} .
$$

Then to have $|\kappa(\pi, \pi)| \leq 1$, we need to have (4.12). 


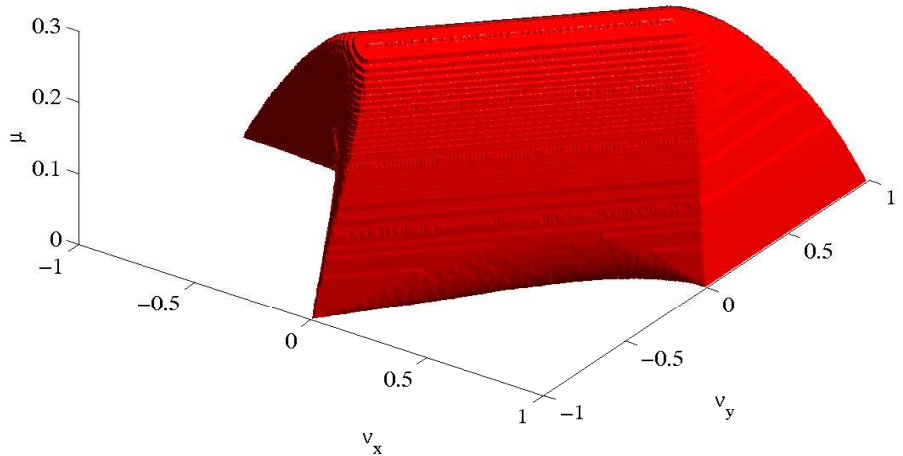

(a)

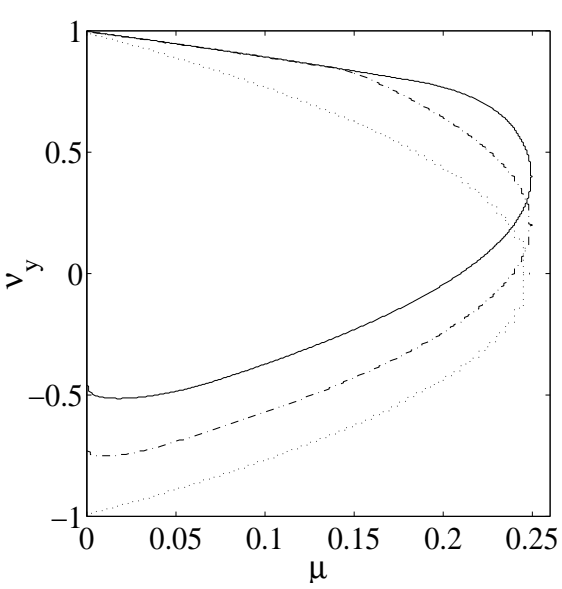

(b)

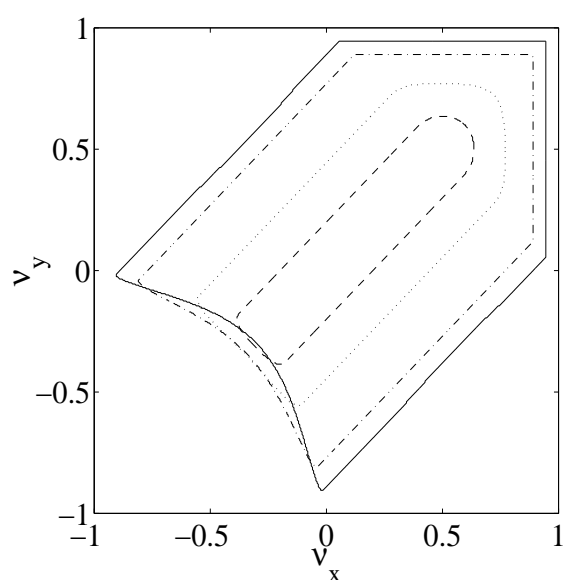

(c)

Figure 8: (a) von Neumann stability analysis for the Polynomial Lax-Wendroff scheme. The scheme is stable inside the shaded region; (b) projection of the figure (a) on the plane $\mu \circ \nu_{y}$ : $\nu_{x}=0(\cdots) ; \nu_{x}=0.2(-\cdot-) ; \nu_{x}=0.4(-) ;\left(\right.$ c) projection of the figure (a) on the plane $\nu_{x} \circ \nu_{y}$ : $\mu=0.05(-) ; \mu=0.1(-\cdot-) ; \mu=0.2(\cdots) ; \mu=0.24(--)$. 
The condition (4.12) is associated with the diagonal lines shown in figure 8c, determining that the stability region is between the two lines as we see in the figure $8 \mathrm{c}$. The condition (4.11) gives us a limit for the diffusion parameter. We observe that this scheme is still stable for simultaneously large values of $\nu_{x}$ and $\nu_{y}$, when $\mu$ is small. Although we plot the stability region for $\left(\nu_{x}, \nu_{y}\right) \in[-1,1] \times[-1,1]$, note that for this scheme we are assuming that both velocities are positive, this is that, $\nu_{x}$ and $\nu_{y}$ are both positive.

The next result is for the Taylor Lax-Wendroff scheme. This scheme was deduced independently of the signs of the velocities.

Lemma 4.7 Necessary conditions for the Taylor Lax-Wendroff scheme (3.3) to be stable are

$$
\begin{aligned}
2\left(\mu_{x}+\mu_{y}\right) & \leq 1 \\
\nu_{x}^{2}+\nu_{y}^{2} & \leq 1-2\left(\mu_{x}+\mu_{y}\right) .
\end{aligned}
$$

Proof: The amplification factor for the Taylor Lax-Wendroff it gives

$$
\begin{aligned}
\left|\kappa\left(\theta_{x}, \theta_{y}\right)\right|^{2}= & {\left[1-\left(\nu_{x}^{2}+2 \mu_{x}\right)\left(1-\cos \theta_{x}\right)-\left(\nu_{y}^{2}+2 \mu_{y}\right)\left(1-\cos \theta_{y}\right)\right.} \\
& \left.-\nu_{x} \nu_{y} \sin \theta_{x} \sin \theta_{y}\right]^{2} \\
& +\left[\nu_{x} \sin \theta_{x}+\nu_{y} \sin \theta_{y}\right]^{2} .
\end{aligned}
$$

For the limiting case $\theta_{x} \rightarrow 0$ and $\theta_{y} \rightarrow 0$ with $\left|\theta_{x}\right| \leq \theta$ and $\left|\theta_{y}\right| \leq \theta$, we can write,

$$
\begin{aligned}
\left|\kappa\left(\theta_{x}, \theta_{y}\right)\right|^{2}= & {\left[1-\left(\nu_{x}^{2}+2 \mu_{x}\right) \frac{\theta_{x}^{2}}{2}-\left(\nu_{y}^{2}+2 \mu_{y}\right) \frac{\theta_{y}^{2}}{2}-\nu_{x} \nu_{y} \theta_{x} \theta_{y}+O\left(\theta^{4}\right)\right]^{2} } \\
& +\left[\left(\nu_{x} \theta_{x}+\nu_{y} \theta_{y}\right)+O\left(\theta^{3}\right)\right]^{2} .
\end{aligned}
$$

After some calculations,

$$
\begin{aligned}
\left|\kappa\left(\theta_{x}, \theta_{y}\right)\right|^{2}= & 1-\left(\nu_{x}^{2}+2 \mu_{x}\right) \theta_{x}^{2}-\left(\nu_{y}^{2}+2 \mu_{y}\right) \theta_{y}^{2}-2 \nu_{x} \nu_{y} \theta_{x} \theta_{y} \\
& +\left(\nu_{x} \theta_{x}+\nu_{y} \theta_{y}\right)^{2}+O\left(\theta^{4}\right) \\
= & 1-\left(2 \mu_{x}+2 \mu_{y}\right)+O\left(\theta^{4}\right) .
\end{aligned}
$$

In order to have $\left|\kappa\left(\theta_{x}, \theta_{y}\right)\right| \leq 1$ for all $\theta_{x}, \theta_{y}$ the condition (4.13) needs to hold. For the particular case $\theta_{x}=\theta_{y}=\pi$ we have,

$$
|\kappa(\pi, \pi)|=\left|1-2\left(\nu_{x}^{2}+2 \mu_{x}\right)-2\left(\nu_{y}^{2}+2 \mu_{y}\right)\right|
$$

and $|\kappa(\pi, \pi)| \leq 1$ is equivalent to (4.14).

This scheme has a smaller region of stability for small $\mu$ compared with the Polynomial Lax-Wendroff scheme (3.1). However this scheme has the advantage that can be used independently of the signs of the velocities. In figure 9 we plot the necessary and sufficient von Neumann stability conditions for the Taylor Lax-Wendroff scheme (3.3). 


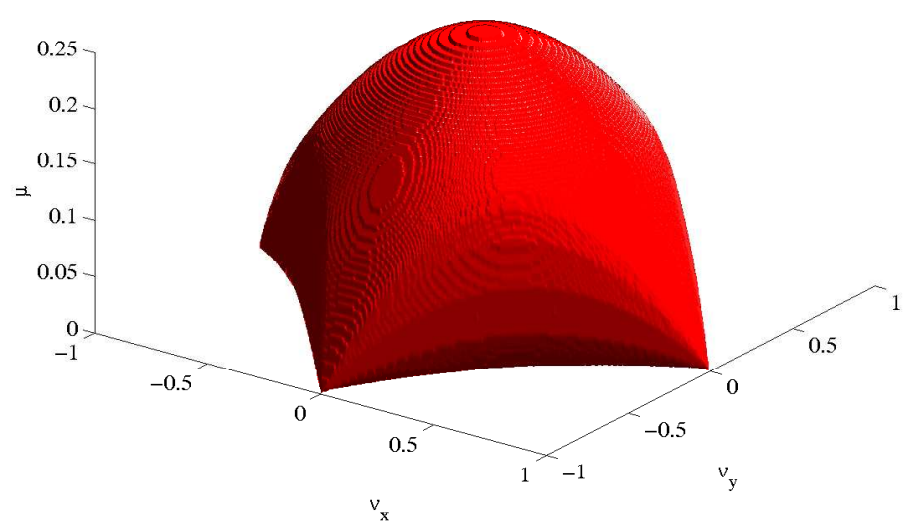

(a)

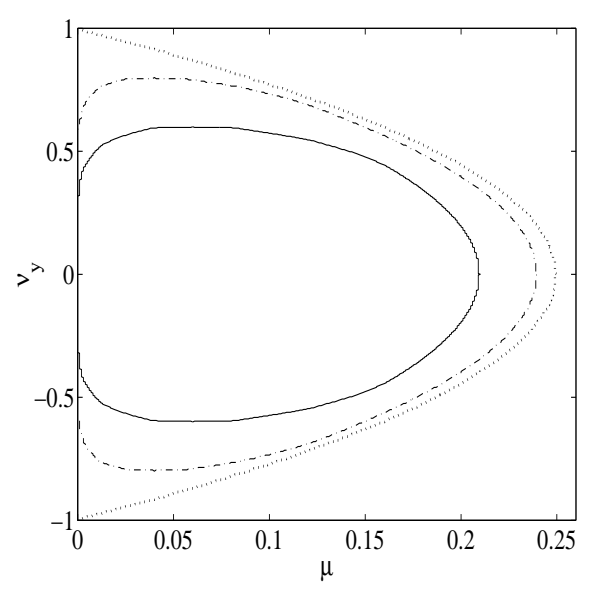

(b)

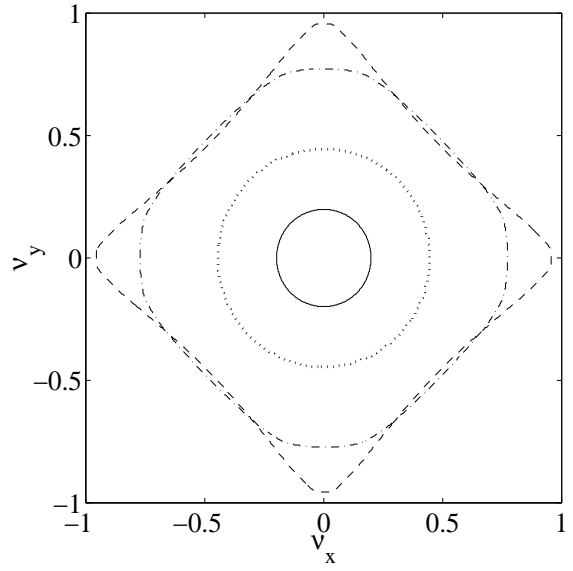

(c)

Figure 9: (a) von Neumann stability analysis for the Taylor Lax-Wendroff scheme. The scheme is stable inside the shaded region; (b) projection of the figure (a) on the plane $\mu \circ \nu_{y}$ : $\nu_{x}=0(\cdots) ; \nu_{x}=0.2(-\cdot-) ; \nu_{x}=0.4(-) ;\left(\right.$ c) projection of the figure (a) on the plane $\nu_{x} \circ \nu_{y}$ : $\mu=0.02(--) ; \mu=0.1(-\cdot-) ; \mu=0.2(\cdots) ; \mu=0.24(-)$. 
Observing the figure 9c, the necessary and sufficient condition for this scheme is the condition (4.14) associated with some other condition that seems to change as $\mu$ increases from $\left|\nu_{x}\right|^{2 / 3}+\left|\nu_{y}\right|^{2 / 3} \leq 1$ to $\left|\nu_{x}\right|+\left|\nu_{y}\right| \leq 1$.

\section{Quickest schemes}

In the one dimensional case we saw that for the Quickest scheme to find analytical sufficient and necessary conditions involves cumbersome expressions that make it difficult to have a clear understanding of the region. In the two dimensional case we should expect to find even more complexity.

We consider first the Polynomial Quickest scheme. The next lemma is about a necessary condition for the stability of this scheme. We plot the sufficient and necessary conditions in figure 10 .

Lemma 4.8 A necessary condition for the Polynomial Quickest scheme (3.2) to be stable is

$$
\begin{aligned}
\left(\nu_{x}^{2}+2 \mu_{x}\right) & \left(1-2 \nu_{y}\right)+\left(\nu_{y}^{2}+2 \mu_{y}\right)\left(1-2 \nu_{x}\right)+2 \nu_{x} \nu_{y} \\
+\frac{2}{3} \nu_{x}\left(1-\nu_{x}^{2}-6 \mu_{x}\right)+\frac{2}{3} \nu_{y}\left(1-\nu_{y}^{2}-6 \mu_{y}\right) & \leq 1
\end{aligned}
$$

Proof: The amplification factor for the method is given by:

$$
\begin{aligned}
\kappa\left(\theta_{x}, \theta_{y}\right)= & 1-\mathrm{i} \nu_{x} \sin \theta_{x}-\mathrm{i} \nu_{y} \sin \theta_{y} \\
& +\left(\nu_{x}^{2}+2 \mu_{x}\right)\left(-1+\cos \theta_{x}\right)+\left(\nu_{y}^{2}+2 \mu_{y}\right)\left(-1+\cos \theta_{y}\right) \\
& -\nu_{x} \nu_{y}\left(\left(1-\cos \theta_{x}\right)\left(1-\cos \theta_{y}\right)+\sin \theta_{x} \sin \theta_{y}\right) \\
& +\frac{1}{6} \nu_{x}\left(1-\nu_{x}^{2}-6 \mu_{x}\right)\left[-2\left(1-\cos \theta_{x}\right)^{2}+2 \mathrm{i} \sin \theta_{x}\left(-1+\cos \theta_{x}\right)\right] \\
& +\frac{1}{6} \nu_{y}\left(1-\nu_{y}^{2}-6 \mu_{y}\right)\left[-2\left(1-\cos \theta_{y}\right)^{2}+2 \mathrm{i} \sin \theta_{y}\left(-1+\cos \theta_{y}\right)\right] \\
& -\nu_{y}\left(\mu_{x}+\frac{1}{2} \nu_{x}^{2}\right)\left[-2\left(1-\cos \theta_{y}\right)\left(1-\cos \theta_{x}\right)\right. \\
& \left.+2 \mathrm{i} \sin \theta_{y}\left(-1+\cos \theta_{x}\right)\right] \\
& -\nu_{x}\left(\mu_{y}+\frac{1}{2} \nu_{y}^{2}\right)\left[-2\left(1-\cos \theta_{x}\right)\left(1-\cos \theta_{y}\right)\right. \\
& \left.+2 \mathrm{i} \sin \theta_{x}\left(-1+\cos \theta_{y}\right)\right] .
\end{aligned}
$$

For the particular case $\theta_{x}=\theta_{y}=\pi$ we have

$$
\begin{aligned}
\kappa(\pi, \pi)= & 1-2\left(\nu_{x}^{2}+2 \mu_{x}\right)-2\left(\nu_{y}^{2}+2 \mu_{y}\right)-4 \nu_{x} \nu_{y} \\
& -\frac{4}{3} \nu_{x}\left(1-\nu_{x}^{2}-6 \mu_{x}\right)-\frac{4}{3} \nu_{y}\left(1-\nu_{y}^{2}-6 \mu_{y}\right) \\
& +4 \nu_{x}\left(2 \mu_{y}+\nu_{y}^{2}\right)+4 \nu_{y}\left(2 \mu_{x}+\nu_{x}^{2}\right)
\end{aligned}
$$

and to have $|\kappa(\pi, \pi)| \leq 1$ we need to have (4.15) . 
We can tell from the complexity of the amplification factor in the previous lemma, that to derive sufficient conditions here is, if not an almost impossible task, then a very hard one.

We notice that on the three dimensional surface displayed in figure 10 , we have $\mu \leq 9 / 16$. This value corresponds to $\left(\nu_{x}, \nu_{y}\right)=(1 / 4,1 / 4)$.

Next we provide a necessary condition for the stability of the Taylor Quickest scheme.

Lemma 4.9 A necessary condition for the Taylor Quickest scheme (3.4) to be stable is

$$
\left(\nu_{x}^{2}+2 \mu_{x}\right)+\left(\nu_{y}^{2}+2 \mu_{y}\right)+\frac{2}{3} \nu_{x}\left(1-\nu_{x}^{2}-6 \mu_{x}\right)+\frac{2}{3} \nu_{y}\left(1-\nu_{y}^{2}-6 \mu_{y}\right) \leq 1
$$

Proof: The amplification factor is given by:

$$
\begin{aligned}
\kappa\left(\theta_{x}, \theta_{y}\right)= & 1-\mathrm{i} \nu_{x} \sin \theta_{x}-\mathrm{i} \nu_{y} \sin \theta_{y} \\
& +\left(\nu_{x}^{2}+2 \mu_{x}\right)\left(-1+\cos \theta_{x}\right)+\left(\nu_{y}^{2}+2 \mu_{y}\right)\left(-1+\cos \theta_{y}\right) \\
& -\nu_{x} \nu_{y} \sin \theta_{x} \sin \theta_{y} \\
& -\frac{1}{3} \nu_{x}\left(1-\nu_{x}^{2}-6 \mu_{x}\right)\left[\left(1-\cos \theta_{x}\right)^{2}+\mathrm{i} \sin \theta_{x}\left(1-\cos \theta_{x}\right)\right] \\
& -\frac{1}{3} \nu_{y}\left(1-\nu_{y}^{2}-6 \mu_{y}\right)\left[\left(1-\cos \theta_{y}\right)^{2}+\mathrm{i} \sin \theta_{y}\left(1-\cos \theta_{y}\right)\right] \\
& \left.-2 \nu_{y}\left(\mu_{x}+\frac{1}{2} \nu_{x}^{2}\right) \mathrm{i} \sin \theta_{y}\left(1-\cos \theta_{x}\right)\right] \\
& \left.-2 \nu_{x}\left(\mu_{y}+\frac{1}{2} \nu_{y}^{2}\right) \mathrm{i} \sin \theta_{x}\left(1-\cos \theta_{y}\right)\right] .
\end{aligned}
$$

For the particular case $\theta_{x}=\theta_{y}=\pi$ we have,

$$
\begin{aligned}
\kappa(\pi, \pi)= & 1-2\left(\nu_{x}^{2}+2 \mu_{x}\right)-2\left(\nu_{y}^{2}+2 \mu_{y}\right) \\
& -\frac{4}{3} \nu_{x}\left(1-\nu_{x}^{2}-6 \mu_{x}\right)-\frac{4}{3} \nu_{y}\left(1-\nu_{y}^{2}-6 \mu_{y}\right) .
\end{aligned}
$$

To have $|\kappa(\pi, \pi)| \leq 1$ we need to have (4.16) .

We plot the sufficient and necessary conditions for the Taylor Quickest scheme in figure 11. By assuming $\mu_{x}=\mu_{y}=\mu$ and $\nu_{x}=\nu_{y}=1 / 4$ in (4.16) we have the condition $\mu \leq 9 / 32$. This is the maximum value that $\mu$ can take inside the stable region (see figure 11).

We turn now to the Quickest scheme suggested by Davis and Moore [2], which is interesting to compare with the Quickest schemes we have devised in this chapter. Their scheme is as follows:

$$
\begin{aligned}
U_{j k}^{n+1}= & U_{j k}^{n}-\nu_{x} \Delta_{x 0} U_{j k}^{n}-\nu_{y} \Delta_{y 0} U_{j k}^{n} \\
& +\left(\frac{1}{2} \nu_{x}^{2}+\mu_{x}\right) \delta_{x}^{2} U_{j k}^{n}+\left(\frac{1}{2} \nu_{y}^{2}+\mu_{y}\right) \delta_{y}^{2} U_{j k}^{n} \\
& +\frac{1}{6} \nu_{x}\left(1-\nu_{x}^{2}-6 \mu_{x}\right) \delta_{x}^{2} \Delta_{x-} U_{j k}^{n}+\frac{1}{6} \nu_{y}\left(1-\nu_{y}^{2}-6 \mu_{y}\right) \delta_{y}^{2} \Delta_{y-} U_{j k}^{n}
\end{aligned}
$$




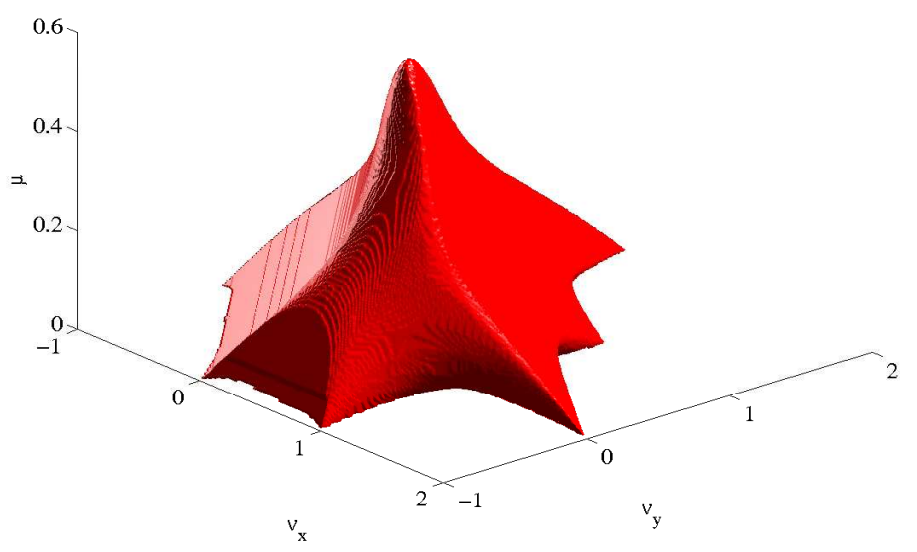

(a)

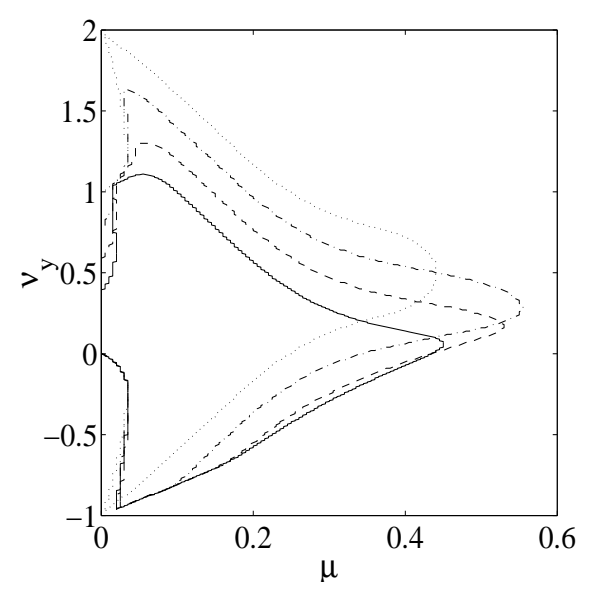

(b)

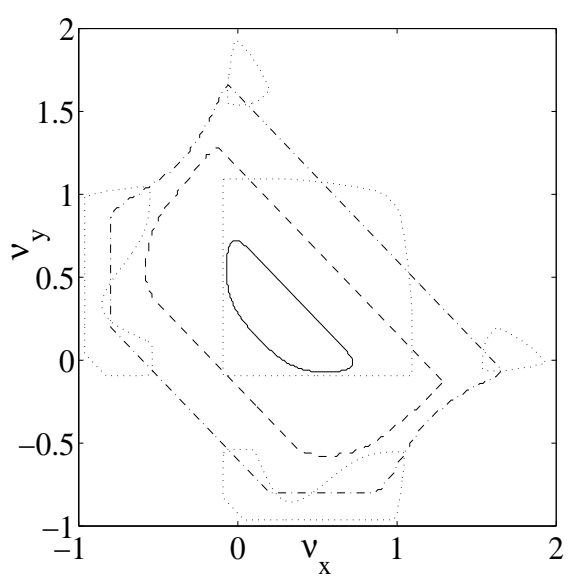

(c)

Figure 10: (a) von Neumann stability analysis for the Polynomial Quickest scheme. The scheme is stable inside the shaded region; (b) projection of the figure (a) on the plane $\mu \circ \nu_{y}$ : $\nu_{x}=0(\cdots) ; \nu_{x}=0.2(-\cdot-) ; \nu_{x}=0.4(--) ; \nu_{x}=0.6(-) ;(\mathrm{c})$ projection of the figure (a) on the plane $\nu_{x} \circ \nu_{y}: \mu=0.02(\cdots) ; \mu=0.1(-\cdot-) ; \mu=0.2(--) ; \mu=0.4(-)$. 


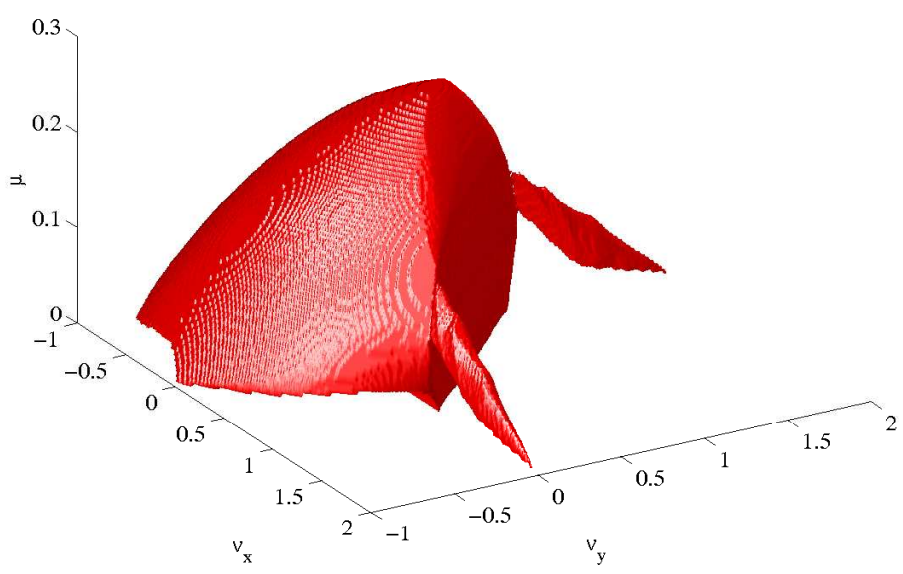

(a)

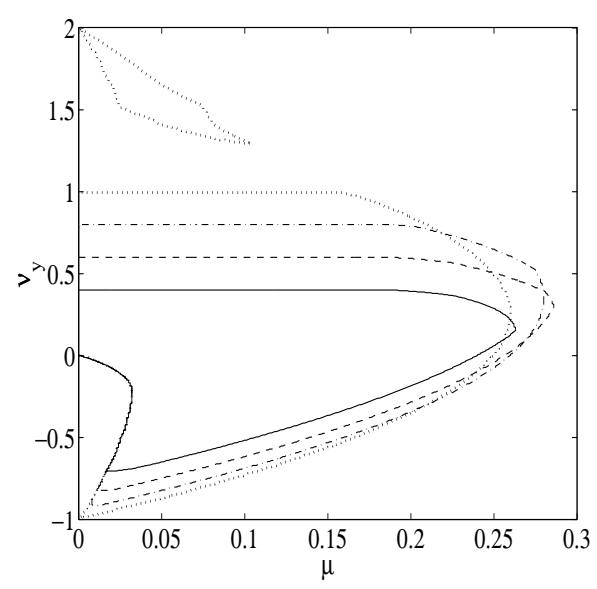

(b)

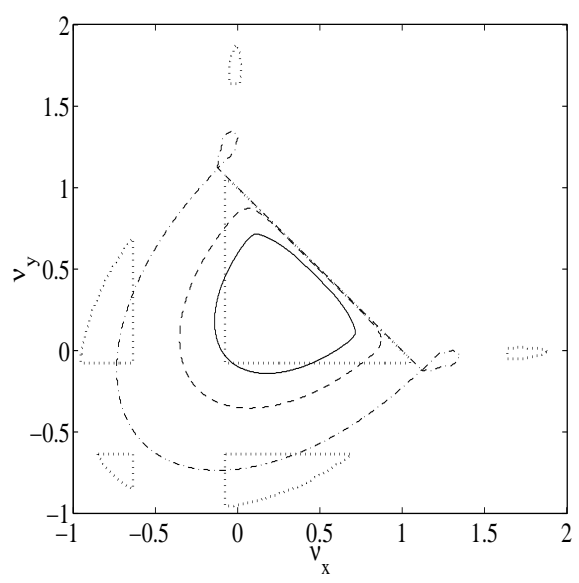

(c)

Figure 11: (a) von Neumann stability analysis for the Taylor Quickest scheme. The scheme is stable inside the shaded region; (b) projection of the figure (a) on the plane $\mu \circ \nu_{y}: \nu_{x}=0(\cdots)$; $\nu_{x}=0.2(-\cdot-) ; \nu_{x}=0.4(--) ; \nu_{x}=0.6(-) ;(\mathrm{c})$ projection of the figure (a) on the plane $\nu_{x} \circ \nu_{y}: \mu=0.02(\cdots) ; \mu=0.1(-\cdot-) ; \mu=0.2(--) ; \mu=0.24(-)$. 


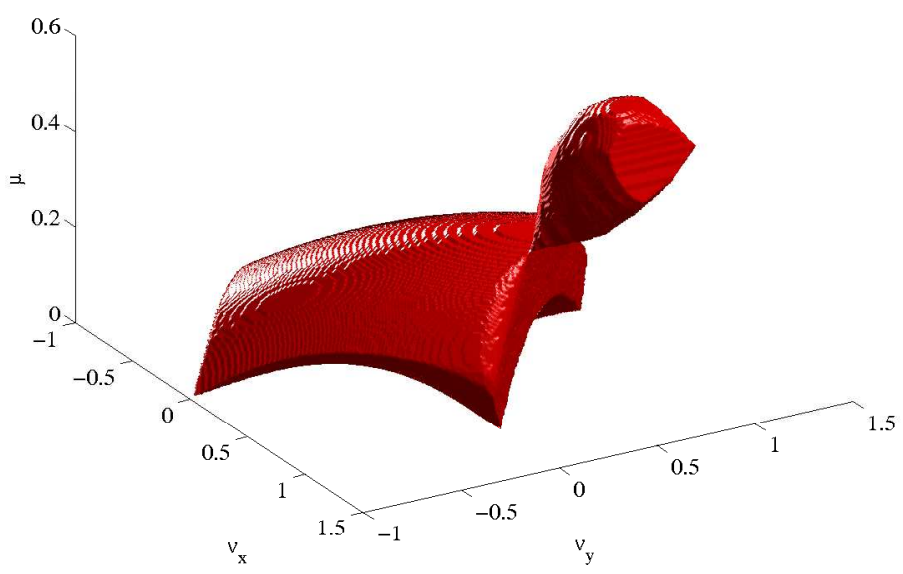

(a)

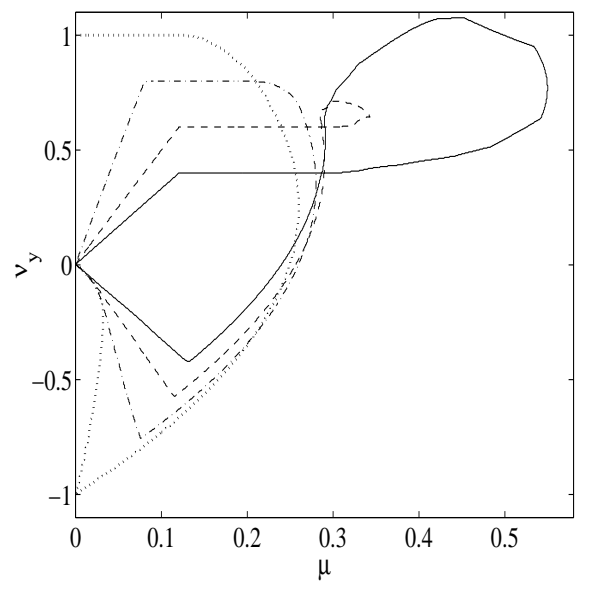

(b)

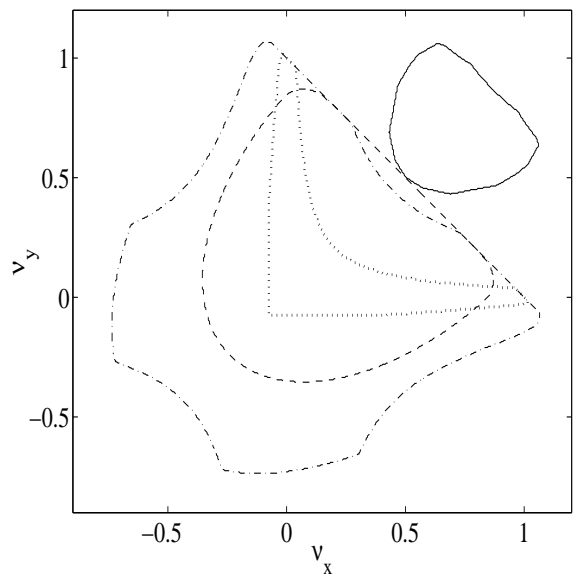

(c)

Figure 12: (a) von Neumann stability analysis for the Davis and Moore Quickest scheme. The scheme is stable inside the shaded region; (b) projection of the figure (a) on the plane $\mu \circ \nu_{y}$ : $\nu_{x}=0(\cdots) ; \nu_{x}=0.2(-\cdot-) ; \nu_{x}=0.4(--) ; \nu_{x}=0.6(-) ;(\mathrm{c})$ projection of the figure (a) on the plane $\nu_{x} \circ \nu_{y}: \mu=0.02(\cdots) ; \mu=0.1(-\cdot-) ; \mu=0.2(--) ; \mu=0.24(-)$. 
Davis and Moore [2] generalised the one dimensional Quickest scheme to two dimensions, although in forming the numerical scheme some $O\left(\Delta t^{2}\right)$ spatial cross derivatives have been omitted to create a simpler algorithm. Formally this reduces the temporal accuracy of the scheme to $O(\Delta t)$. Consequently Davis and Moore employed small time-steps to minimise the error associated with the neglected $O\left(\Delta t^{2}\right)$ term.

We analyse the stability for this scheme using the von Neumann analysis in the same way as for the previous Quickest schemes. To find a necessary condition for the Davis and Moore Quickest scheme, we evaluate the amplification factor for the phase angles of high frequency $\theta_{x}=\theta_{y}=\pi$. In that way we obtain the same necessary condition as for the Taylor Quickest scheme, given by (11). The stability region for this scheme is plotted in figure 12. According to this figure it seems that another necessary condition for stability is that for $\mu \leq 1 / 4$, we need to have $\nu_{x}+\nu_{y} \leq 1$. For small $\mu$ the stable region seems to be quite small, compared with the previously studied schemes.

\section{Summary}

In this paper we provided von Neumann stability regions for various finite difference schemes. The Lax-Wendroff schemes considered present regions of stability that are sufficient and necessary conditions, with a shape that appears to have a simple form, although we found considerable difficulties when we attempted to find these conditions analytically. The main source of these difficulties was related to the majoration of the Fourier terms associated with the mixed derivatives. The Quickest schemes present more awkward regions and they do not seem to show an obvious regularity leading us to conjecture that to provide the analytical sufficient and necessary conditions is an extremely difficult task. Therefore we provide only the analytical necessary conditions, although sufficient and necessary regions of stability are calculated numerically.

We know that the presence of boundary conditions interferes with the stability of a finite difference scheme. Although in the presence of boundaries that are not periodic the von Neumann condition is no longer a sufficient condition, it remains an important necessary condition.

\section{Ackowledgments}

ES acknowledges support from Sub-Programa Ciência e Tecnologia do 2 Quadro Comunitário de Apoio and Coimbra University, Portugal.

\section{References}

[1] Beckers, J.M. (1992). Analytical linear numerical stability conditions for an anisotropic three-dimensional advection-diffusion equation. SIAM Journal of numerical analysis 29, 701-713.

[2] Davis, R.W. and E.F. Moore (1982). A numerical study of vortex shedding from rectangles. Journal of Fluid Mechanics 116, 475-506. 
[3] Gustafsson, B., H.O. Kreiss and J. Oliger (1995). Time-dependent problems and difference methods. Wiley-Interscience.

[4] Hindmarsh, A.C., P.M. Gresho and D.F. Griffiths (1984). The stability of explicit Euler time-integration for certain finite difference approximations of the multidimensional advection-diffusion equation. International journal for numerical methods in fluids 4, 853-897.

[5] Kwok, Y-K and K-K Tam (1993). Stability analysis of three-level difference schemes for initial-boundary problems for multidimensional convective-diffusion equations. Communications in Numerical methods in engineering 9, 595-605.

[6] Lax, P.D. and B. Wendroff (1964). Difference schemes for hyperbolic equations with high order of accuracy. Communications on Pure and Applied Mathematics 17, 381398.

[7] Leonard, B.P. (1979). A stable and accurate convective modelling procedure based on quadratic upstream interpolation. Computer methods in applied mechanics and engineering 19, 59-98.

[8] Leonard, B.P. (1980). Note on the von Neumann stability of the explicit FTCS convection diffusion equation. Applied Mathematical Modeling 4, 401-402.

[9] Morton, K.W and I.J Sobey (1993). Discretization of a convection-diffusion equation. IMA Journal of numerical analysis 13, 141-160.

[10] Morton, K.W. (1996). Numerical Solution of Convection-Diffusion Problems, Chapman and Hall: London.

[11] Richtmyer, R.D. and K.W. Morton (1967). Difference methods for initial-value problems. Wiley-Interscience: New York.

[12] Siemieniuch, J. and I. Gladwell (1978). Analysis of explicit difference methods for the diffusion-convection equation. International Journal for numerical methods in engineering 12, 899-916.

[13] Turkel, E. (1977). Symmetric Hyperbolic difference schemes and Matrix Prolems. Linear Algebra and its applications 16, 109-129.

[14] Wesseling, P.(1996). Von Neumann stability conditions for the convection-diffusion equation. IMA Journal of numerical analysis 16, 583-598.

[15] Verwer, J.G. and Sommeijer (1997). Stability analysis of an odd-even-line hopscotch method for three-dimensional advection-diffusion problems. SIAM Journal of numerical analysis 34, 376-388. 